\title{
Some experiments in the gestural control of synthesized sonic textures
}

\author{
Daniel Arfib ${ }^{1}$, Jean-Michel Couturier ${ }^{1}$, and Jehan-Julien Filatriau ${ }^{1,2}$ \\ 1 CNRS-LMA, 31 chemin Joseph Aiguier \\ 13402 Marseille Cedex 20, France \\ \{arfib, couturier\}@lma.cnrs-mrs.fr, \\ 2 Laboratoire de Télécommunications et Télédétection, \\ Université Catholique de Louvain (UCL), Belgium \\ filatriau@tele.ucl.ac.be
}

\begin{abstract}
In this paper, we introduce some exploratory ideas and applications involving the gestural control of sonic textures. Three examples of how the gestural control of synthesized textures can be implemented are presented: scratching textures, based on the gesturalized exploration of a visual space; dynamic noise filtering, where gestures influence a virtual slowly moving string used to filter a noise; and breathing textures, where the metaphor of breathing is used in the sound as well as in the gestural control. Lastly, we discuss how to find connexions between appropriate gestures and sonic texture processes, with a view to producing coherent and expressive digital musical instruments.
\end{abstract}

\section{Sonic textures and gestural control}

Sonic textures are characterized by both microscopic and macroscopic features: on the short term scale, they are composed of a series of microstructural components which are subject to some randomness; whereas on the long term scale, some temporal and spectral cohesion is preserved. Sonic textures can result either from a process of computer analysis and synthesis or from synthesis alone [3]; the three examples we present in this paper are of the latter kind. Sound synthesis can be controlled by gestures using sensors connected to a computer. The link between gestural data and the synthesis parameters is called "Mapping" [1]. It is only when the gesture is properly linked to the sonic process, not only technically but especially at the human emotional level, that one can speak of a digital musical instrument.

\section{Gestural control of sonic textures : some examples}

\subsection{Scratching textures}

This digital instrument prototype is based on the gesturalized exploration of a visual space. It involves the real-time implementation of the Functional Iteration 
Synthesis (FIS) [3] driven by a bi-manual gestural control using a tablet-screen and a joystick. FIS is an algorithm resulting from the wave terrain synthesis [6], where the terrains are obtained by iterating non-linear functions. An orbit is then traced on the three-dimensional surface to generate a waveform corresponding to the variations in the elevation of the trajectory over the terrain.
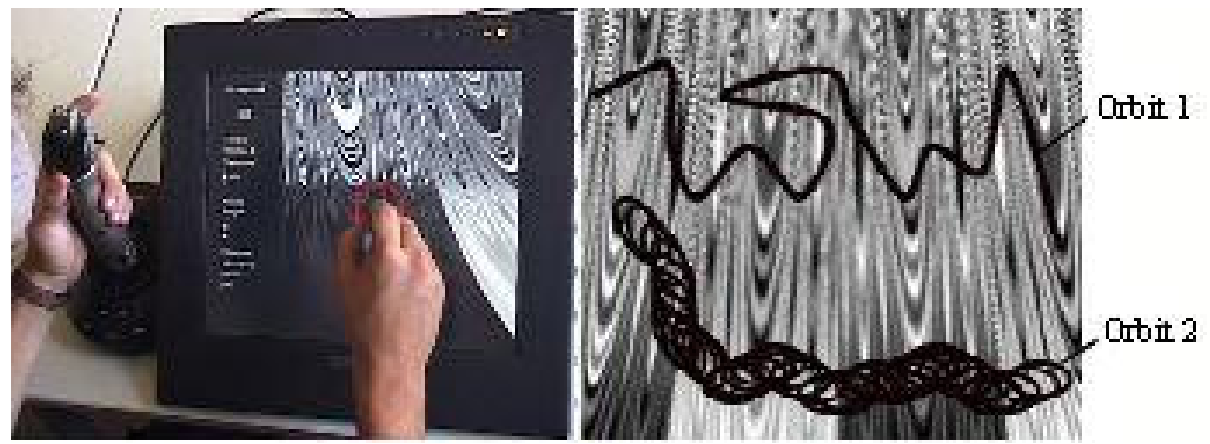

Fig. 1. Left: the scratched gestural control using a tablet and a joystick. Right, two orbits created by direct control (orbit 1) and by parametric control (orbit 2).

A gestural control inspired from the surface scratching allegory was developed [7]. The exploration of the wave terrains is carried out here either by performing linear trajectories (using the direct mode) or looping trajectories (using the parametric mode) (Fig. 2.1). In the direct mode, the orbit corresponds to the actual trajectory drawn by the user on the tablet screen, and the spectral features of the texture depend directly on the performers hand movements. In the parametric mode, trajectories are generated via three control parameters: the center of the trajectory drawn by the pen on the tablet, and its radius and its velocity, both of which can be modulated via a joystick. This parametric control makes it possible to overcome the limitations inherent to direct control and to create pseudo-pitched sonic textures.

\subsection{Dynamic noise filtering}

In the Filtering String instrument, gestures are used to control a virtual slowly moving string (based on a spring-mass model), which is used to filter a noise [2]. The string shape drives the gains of 32 filters and is displayed on a screen; the string model controls both the sound and the graphics. The choice of resonant frequencies and the quality factors (linked to the bandwith) of the filters give the sound a basic color; the motion of the string adds fluctuations to the sound. With this technique, it is possible to create a texture with complex but natural variations, because the physical behaviour of the string is well known. The user acts on the sound fluctuations indirectly, by interacting with the dynamic string 

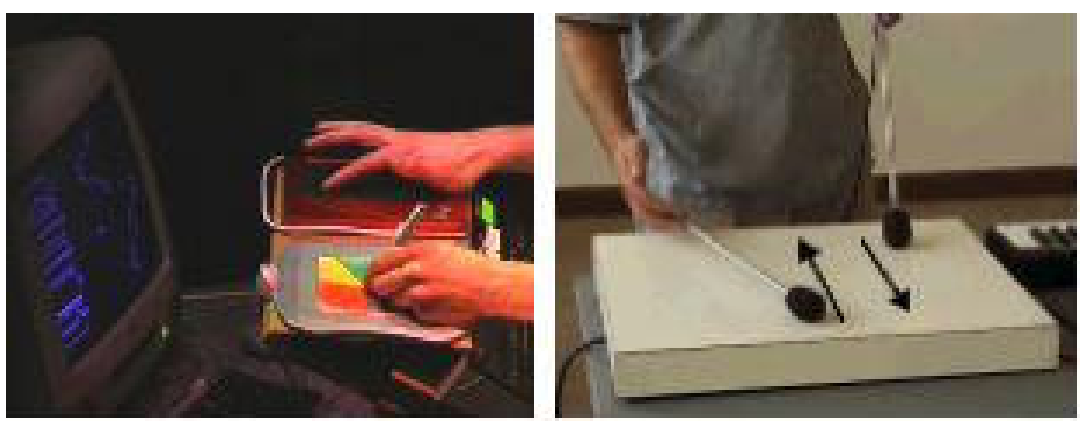

Fig. 2. Left: the gestural control of a filtering string. Right: an alternating gesture linked to breathing textures.

using a graphic tablet and a multi-touch surface (Fig. 2.2 left). The position and pressure of the stylet on the graphic tablet affect the sonic texture by changing the intrinsic properties of the string (such as its tension, stiffness, and damping). Forces can be applied to the virtual string by exerting pressure on the multitouch surface; this changes the strings equilibrium position and thus modifies the frequency spectrum of the texture. With one hand (using the stylet), the user configures the dynamic system and thus determines how the string will respond to the other hand gestures (on the touch surface). The effects of the touch pad gestures on the sound will depend on the string configuration. For example, at low stiffness values, the string will move slowly and will not respond to fast movements on the touch pad: in this case, fast gestures are filtered by the dynamic string. With his hands, the user can impart energy on the string and change the way it moves; the sonic texture will evolve, although it keeps its identity.

\subsection{Breathing textures}

Playing this instrument requires making ecological hand gestures with an alternating pattern, which interact with a breathing sound production process. Windy textures can be produced by multiplying a band-limited noise signal by a sine wave (amplitude modulation) [5]. In this way, the band-limited spectrum is translated by the sine wave frequency value, giving rise to the perceptual sensation of a definite pitch, while the timbre is noisy but quite smooth. The control parameters are the perceptual pitch, the noisiness, and the amplitude of the sound. The metaphor of breathing (inhaling and exhaling) can be used, for example, by alternating two different sonic textures, which can also evolve with time. The most natural way of gesturalizing this metaphor is to use bimanual gestures, with the two hands moving in opposite directions (Fig. 2.2 right). Generally speaking, it can be appropriate to use familiar gestures (which are sometimes called ecological gestures) mimicking common manual activities and to link them to these sounds. 


\section{$3 \quad$ Textures and gestures}

Sonic textures are specific: they are perceived as sound masses with a rather indefinite pitch, and the usual attack part is often replaced by a series of transients initiated throughout the duration of the sound. Another aspect of sonic textures links up with the fact that many natural sounds are textures resulting from the movements of bodies, as well as those of fluids and gases. It is therefore possible to set up a relationship between the energy of the performers movements and the evolution of the sound (see an earlier study by Hunt [4]). The best way of determining which gestures should be used to control a texture is to create a mental image of the texture before trying to find the most appropriate gestures for controlling it, rather than looking first for the most efficient artificial links.

\section{Conclusion}

We have established that it is possible to create new digital instruments using sonic textures, taking their temporal and spectral specificities into account. We have concluded from our experiments that the gestures associated with the production of sonic textures should correspond to the ecological nature of these sounds, and that the gestural control is at least as important as the synthesis algorithm. We intend in the future to investigate the most suitable ecological gestures for producing ecological sounds, paying due attention to the specificities of sonic textures. Potential applications such as the musical possibilities of these instruments or the emotional textural rendering will also be investigated.

\section{References}

1. Arfib, D., Couturier, J.M. \& Kessous, L., "Design and Use of Some Digital Musical Instruments", in A. Camurri \& G. Volpe (Ed) Gesture-Based Communications in Human-Computer Interaction, Lectures notes in Artificial Intelligence, LNAI 2915, pp. 509-518. Springer Verlag, 2004.

2. Arfib, D., Couturier, J.M. \& Kessous, L., "Gestural Strategies for specific filtering processes", in Proceedings of Digital Audio Effects 2002 conference (DAFx02, pp. 1-6, Hamburg, Germany, 26-28 sept 2002.

3. Di Scipio A., "Synthesis of environmental sound textures by iterated non linear functions and its ecological relevance to perceptual modelling", Journal of New Music Research, Vol. 31 p. 109-117, 2002.

4. Hunt A., Kirk R., Mapping Strategies for Musical Performance, Trends in Gestural Control of Music, CD-rom, Edited by M. Wanderley and M. Battier, Ircam, 2000.

5. Mathews Max V., The Technology of Computer Music, MIT Press, Cambridge, MA, 1989.

6. Roads C., The Computer Music Tutorial, MIT Press, Cambridge, MA, 1996.

7. Wessel D., Wright M., Schott J., Intimate Musical Control of Computers with a Variety of Controllers and Gesture Mapping Metaphors, in Proceedings of the New Instruments for Musical Expression conference (NIME02), Dublin, Ireland, May 24-26, 2002. 